\title{
Multi-frequency Technique for Frequency Response Measurement and its Application to Servo System with Friction
}

\author{
Ehsan Keikha*, A. Al Mamun ${ }^{\diamond}$ T. H. Lee ${ }^{\square}$, C. S. Bhatia \\ Department of Electrical \& Computer Engineering \\ National University of Singapore (Tel: 65-651-62251) \\ E-mail: $\left\{{ }^{*} \mathrm{~g} 0800472, \diamond\right.$ eleaam, eleleeth, ^elebcs $\} @$ nus.edu.sg
}

\begin{abstract}
In this paper, the effect of nonlinear distortion on system identification is studied. In particular, the effect of nonlinear friction on frequency response measurement is examined. Design of multifrequency excitation signal that minimizes nonlinear effect on frequency response measurement has been explored and the best linear approximation model has been defined. An identification method using differential No Interharmonic Distortion (NID) excitation with optimized crest factor is proposed. The method is used to identify the best linear model of a hard disk drive actuator.
\end{abstract}

Keywords: System identification, frequency response, friction model, best linear approximation

\section{INTRODUCTION}

Ever growing demand for higher capacity of information storage caused an increase in the areal density of hard disk drives (HDD) by $40 \%$ - 60\% every year. Recording density in commercially available HDDs still lies below 1 Tbits/in ${ }^{2}$ but the next milestone is predicted to be $10 \mathrm{Tbits} / \mathrm{in}^{2}$. Assuming bit aspect ratio (bit length : bit width) of $2: 1$, track density of about 2,200,000 tracks per inch (TPI) will be required to achieve such density, which translates into track pitch of 11.6 $\mathrm{nm}$. Desired tracking error in positioning the read/write head above a track on rotating disk for reading/ writing data is less than $10 \%$ of track pitch. So $3 \sigma$ track miss-registration (TMR) budget is less than $1.16 \mathrm{~nm}$ in $10 \mathrm{Tbits} / \mathrm{in}^{2}$ recording. Improving technological edge in servo mechanical control in terms of theoretical concept and engineering development is essential in reaching the TMR target of that scale. Modern approaches, e.g., robust multi-rate control (Nagamune et al., 2010), have been suggested to achieve this future goal; but uncertainties and plant nonlinearity remain as obstacles.

Pivot friction in HDD's voice coil motor (VCM) actuator is modelled as a nonlinear function of velocity shown in Figure 1 which includes stiction $\left(F_{S}\right)$, Coulomb friction $\left(F_{c}\right)$ and viscous friction. This static friction model fails to capture observed nonlinear phenomena such as hysteresis, position dependence, and variations in the breakaway. In nanopositioning servo systems, friction adversely affects settling time, overshoot and steady state error. Modelling pivot friction has therefore been a subject of interest in industries as well as among academia. Researchers have also investigated for models that represent dynamic behaviour of friction.

Pivot friction was studied by (Abramovitch et al., 1994) and (Feei Wang et al. 1994), who presented their findings in both time and frequency domains. In frequency domain, friction causes reduced gain at low frequency. It is also shown that low frequency response varies with amplitude of excitation signal used to measure the frequency response. This effect becomes an obstacle when the input current is reduced to a minimum, suggesting the possibility of hindrances to an efficient control of the actuator.

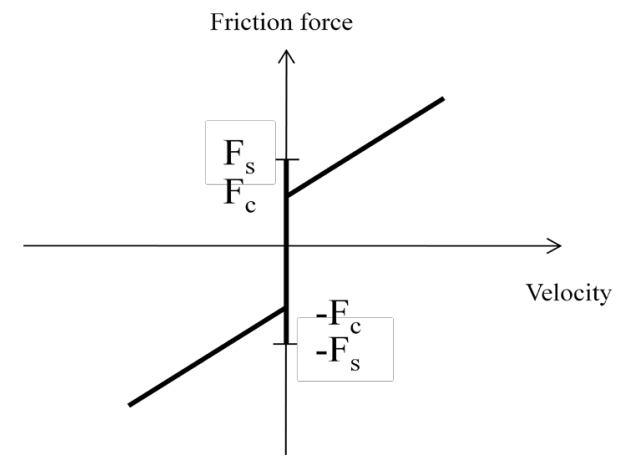

Figure 1: Relation between friction and velocity

Frequency response is widely used by practicing engineers to understand system behaviour. However, identification of model from the frequency response of a nonlinear system produces a linearized model at given operating condition. So care is required while identifying linear model; modelling of nonlinearity is also necessary. (Byun et al. 1997) proposed a method for precise identification of friction parameters. (Kemao Peng et al. 2005) obtained a model of the VCM actuator including friction and nonlinear characteristics through examination of the configuration and structure of the actual system and analysis of its physical effects together with its responses in time domain and frequency domain.

Frequency domain identification techniques use experimental data to estimate a Frequency Response Function (FRF) from which model parameters are obtained. Unfortunately, frequency response is applicable to linear systems only. 
Experimentally observed frequency response of a nonlinear system essentially produces the result for the system linearized around the specific operating point, ignoring the existence of nonlinearity. FRF measured using swept sine excitation (Sherif and Bassioni, 1994) or random noise excitation (Hensen et al., 2002) results in such approximate linear model.

Nonlinear effect can be reduced by measuring frequency response using a single-frequency sine, one frequency at a time (Symens et al., 2002) by gradually sweeping through the desired range of frequencies. But the process is timeconsuming - a problem that can be overcome by applying an excitation signal constructed using all frequencies of interest. Use of such input allows us to measure the response in one pass. This method, known as multi-frequency system identification (Yung-Yaw Chen et al. 2002, Mueller et al. 1996), suffers from nonlinear distortion.

In case of a linear system, the frequency components at the output are same as those at the input; change takes place only in magnitude and phase. However, response of a nonlinear system to a single frequency sinusoidal excitation contains input frequency and its harmonics. The problem becomes worse when multi-frequency input is used, not only will harmonics be produced, but inter-modulation components of harmonics will also be generated. Superposition of various frequency components distorts the measured frequency response. Use of non-interfering frequencies to generate input excitation can resolve this issue (Evans and Rees, 2000, Mueller et al. 1996). Proper scaling of amplitude for each frequency component leads to crest factor optimization problem (Solomou et al. 2002)(Guillaume et al. 1991).

Main objective of this paper is to propose a new multifrequency identification method to estimate the best approximation of frequency response function (FRF) of the underlying linear system. To reach this ultimate goal it is of great importance to ensure that the frequency response is not dependent on the magnitude of the excitation signal. The method has been tested for identification of linear model of a servo actuator with friction. The method can easily be extended to estimate the coefficient of Coulomb friction; however, it may not be true for other nonlinearity.

\section{DISTORTION DUE TO NONLINEARITIES}

Consider a linear time invariant system described by the impulse response model of the following form

$$
y(t)=\int_{0}^{T_{S}} u(t-\tau) h(\tau) d \tau+n(t)
$$

where $u(t), y(t), n(t)$ are input, output, and noise, respectively, and $h(\tau)$ is the impulse response of the system. The frequency response function (FRF) $H(j \omega)$ can be estimated as $Y(j \omega) / U(j \omega)$ where $Y(j \omega)$ and $U(j \omega)$ are the Fourier transforms of output and input, respectively. Consider a multi-sine signal consisting of $N_{u}$ distinct harmonic $\left\{\omega_{u}\right\}$,

$$
u(t)=\sum_{u=1}^{N_{u}} a_{u} \cos \left(\omega_{u} t+\alpha_{u}\right)
$$

where $\omega_{u}=\frac{2 \pi k_{u}}{T}$ and $k_{u}$ are monotonically increasing harmonic numbers $\left(k_{u} \in \mathbb{N}, u=1,2, \cdots N u\right)$ and $\left\{a_{u}\right\}$ is the specified amplitude spectrum. One advantage of using a multi-frequency signal is that as it is periodic, it can be easily generated for any given arbitrary spectrum. The steady state output of the system when subjected to input (2),

$$
y(t)=\sum_{u=1}^{N_{u}} a_{u}\left|\mathrm{G}\left(\mathrm{j} \omega_{\mathrm{u}}\right)\right| \cos \left(\omega_{u} t+\alpha_{u}+\phi_{u}\right)
$$

The linear convolution integral (1) can be generalized to describe a general, causal, time invariant, stable nonlinear system -

$$
y(t)=\sum_{i=1}^{\infty} \int h_{i}\left(\tau_{1}, \ldots, \tau_{i}\right) \prod_{j=1}^{i} u\left(t-\tau_{i}\right) d \tau_{j}
$$

where $h_{n}\left(t_{1}, \ldots, t_{n}\right)$ is the $\mathrm{n}$ dimensional $\mathrm{n}^{\text {th }}$ order Volterra kernel and can be viewed as an $\mathrm{n}$ dimensional impulse response. The equivalent frequency domain representation of such system is

$$
Y\left(s_{1}, s_{2}, \ldots, s_{n}\right)=\sum_{i=1}^{n} H_{i}\left(s_{1}, \ldots, s_{i}\right) \prod_{j=1}^{i} S\left(s_{j}\right)
$$

where $H_{n}\left(s_{1}, \ldots, s_{n}\right)$ is the corresponding $\mathrm{n}$ dimensional Laplace transform of $h_{n}\left(t_{1}, t_{2}, \ldots, t_{n}\right)$. When a multi-freq signal (eq 2) is applied to such system, the output signal contains input frequencies as well as their harmonics. The frequency contributions due to the nonlinearities can be divided into two types - harmonic contribution generated from pairs of equal positive and negative frequency or another input frequency with pair of equal pair of frequency (Type I) and inter-harmonic contribution not accounted for by Type I contributions (Type II). Even order nonlinearities can be suppressed by considering only odd harmonics, as the nonlinear contributions of the even order effects will fall at even harmonics (Solomou et al., 2002). As a result they will be distinct from the linear contributions as well as higher odd order one. Let's consider, for illustrative purposes, a simple cubic static nonlinearity with unit gain. The nonlinear contribution of the output corresponding to a multi-frequency input can be expressed in the frequency domain as

$$
\begin{aligned}
& Y_{n l}(j \omega)=\sum_{\substack{m=-N_{u} \\
m \neq 0}}^{N_{u}} \sum_{\substack{m=-N_{u} \\
n \neq 0}}^{N_{u}} \sum_{\substack{m=-N_{u} \\
o \neq 0}}^{N_{u}}\left[a_{m} a_{n} a_{o}\right] \\
& \times \exp \left(j\left(\alpha_{1}+\alpha_{2}+\alpha_{3}\right)\right) \\
& \times \delta\left(\omega-\frac{\left(k_{m}+k_{n}+k_{o}\right) 2 \pi}{T}\right)
\end{aligned}
$$

The extent of distortion due to nonlinearity at the test frequencies can be quantified as

$$
E=\frac{1}{N_{u}} \sum_{u=1}^{N_{u}}\left|Y_{n l}\left(j \frac{2 \pi k_{u}}{T}\right)\right|
$$


If the primary aim of the identification experiment is to identify the linear kernels accurately, it is possible to reduce the contribution of the nonlinearities by a suitable choice of phases. Thus, from an optimization point of view, one requires to choose phases of input multi-sine signal such that the input crest factor and nonlinear contributions at test frequencies are minimized.

\section{Best Linear Approximation for friction}

The best linear approximation of nonlinear system is defined as the optimal model which minimizes the mean square error between the true output and the model output for a particular class of stochastic inputs:

$$
G_{B L A}=\arg _{G} \min E\left[(y(t)-G(q) u(t))^{2}\right]
$$

with $\mathrm{u}(\mathrm{t})$ and $\mathrm{y}(\mathrm{t})$ the measured input and output respectively, and $G(q)$ the linear transfer function model where $q$ is the shift operator. With this definition the best linear approximation for a Wiener-Hammerstein (WH) system shown in Figure (2) is

$$
G_{B L A}=\eta G_{1}(\omega) G_{2}(\omega)
$$

where $\eta$, the best linear approximation of the static nonlinearity, is a scaling factor that depends on the power spectrum of (rms value and colouring) (J. Schoukens et al 2005a).

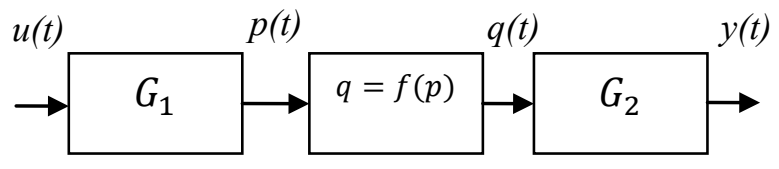

Figure 2: Wiener-Hammerstein system.

In nonlinear systems with a Wiener-Hammerstein structure in the feedback loop(WH-NLFB), it can be shown that changes of the input signal (rms and colouring) lead to shape changes of both the amplitude and phase of $\mathrm{G}_{\mathrm{BLA}}$ (J. Schoukens et al 2005a). This corresponds to a pole displacement, which is consistent with system and control theory. The best linear approximation of such system is:

$$
G_{B L A}=\frac{G_{0}(\omega)}{1+\eta G_{1}(\omega) G_{2}(\omega) G_{0}(\omega)}
$$

where $G_{0}(\omega)$ is the linear system in feedforward and $\mathrm{WH}$ nonlinear system is located in feedback loop.

\subsection{Decomposition of servo system with Friction}

The plant in Figure 3(a) is a nonlinear system with friction. It can be decomposed into a WH-NLFB as friction force can be treated as a nonlinear negative feedback from the output velocity, shown in Figure 3(b), where $G_{0}(s)$ is a linear transfer function. Nonlinearity in the feedback path represents $F_{f}(t)$ described in Figure 1. Taking linear viscous friction as part of the linear system, the nonlinear plant description reduces to the one shown in Figure 3(c).
From Figure 3(c), $w$ and $v$ are input and output, respectively, of the linear block. It is possible to identify the linear model from the signals $w$ and $v$. However, the input $w$ to the linear block is not measureable. Signals $w$ and $u$ are different due to nonlinear distortion. Linear system identification using $u$ and $v$ does not give a satisfactory model.

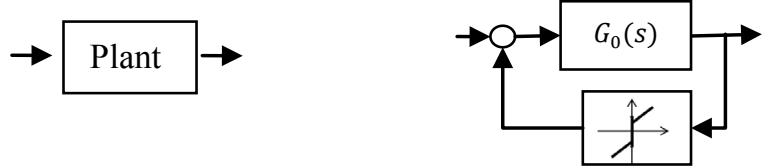

(a)

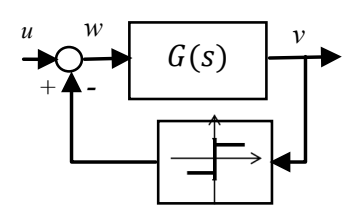

(b)

(c)

Figure 3: Decomposition of servo system with friction

\subsection{Responce with Difference Signal}

(Yung-Yaw Chen et al. 2002) proposed a novel method to perform system identification for a class of nonlinear system shown in Figure 3(c) which was based on square wave excitation. Although using difference mapping in the input and output can minimize the effect of nonlinearity, use of square wave excitation produces many interfering harmonics which degrade the accuracy of estimated model.

Let's assume $u_{1}$ and $v_{1}$ as input and output of the system shown in Figure 3(c) and $w_{1}$ is the input of the linear block. If $u_{2}$ is a perturbed version of the input which corresponds to $v_{2}$ and $w_{2}$, we can say, due to linear mapping between $w$ to $v$, that

$$
w_{1}-w_{2} \rightarrow v_{1}-v_{2}
$$

$w_{1}-w_{2} \rightarrow v_{1}-v_{2}$
We use a simulated nonlinear plant, Assumed $G_{0}=\frac{10}{s+100}$ and $F_{c}=0.2$, to demonstrate the effectiveness of using difference signal to identify WH-NLFB model. Simulated results for an input sine wave of amplitude 2 and frequency $10 \mathrm{~Hz}$ are shown in Figure 4.

In Figure 4(a), the dotted line represents the input signal $u$, the dash-dot line the velocity $\mathrm{v}$, and the solid line input $\mathrm{w}$, input to linear block. The difference between the inputs $\mathrm{u}$ is shown in Figure 4(b) and the difference between the inputs of the linear block $\mathrm{w}$ is shown in Figures 4(c) and 4(d). Two difference signals appear to be very similar except for the small glitches. It is shown by (Yung-Yaw Chen et al. 2002) that the glitches become smaller with increasing input amplitude [Figure 4(d)].

It is clear from Figure $4(\mathrm{c}, \mathrm{d})$ that $\left(\mathrm{u}_{1}-\mathrm{u}_{2}\right)$ can be made almost identical to $\left(w_{1}-w_{2}\right)$ by properly choosing $u_{1}$ and $u_{2}$. Taking $\left(u_{1}-u_{2}\right)$ and $\left(v_{1}-v_{2}\right)$ as the input/output pairs, which is equivalent to input/output pairs $\left(\mathrm{w}_{1}-\mathrm{w}_{2}\right)$ and $\left(v_{1}-v_{2}\right)$, the linear block of the target system can be identified using a number of identification chemes. 


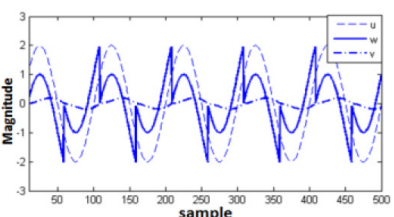

(a)

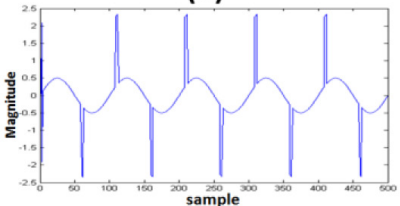

(C)

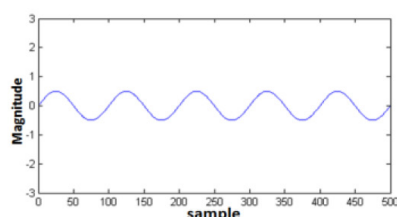

(b)

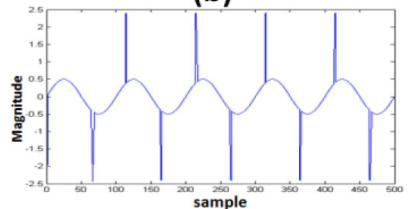

(d)
Figure 4: Simulated result.

In addition, as the output of the nonlinear block in Figure 3(c) is not dependent on input magnitude, it is obvious that, for very large amplitude of input excitation, $u$ and $w$ are identical. In other words, the best linear approximation of the system is $\frac{v}{u}$ if the $u \rightarrow \infty$. But it is not possible in real world; so design of a practical method to identify the best approximation of the nonlinear system if of great importance. We start with the issue of designing input excitation followed by suitable identification algorithm.

\section{MULTIFREQUENCY TEST SIGNAL DESIGN}

A wide variety of test signals is available for system identification. The aim of this section is to design a multi-sine signal with minimum harmonic contribution (Type I) at the output to minimize the effect of nonlinear distortion due to superposition of harmonic frequencies. Consider a multi-sine signal which consists of $N_{u}$ distinct harmonic $\left\{\omega_{u}\right\}$ as shown in (2). If this input is applied no a nonlinear system, harmonics and inter-modulation harmonics will corrupt the measured frequency response. (Yung-Yaw Chen et al. 2002) used harmonics that are prime number multiples of the fundamental frequency to reduce this distortion.

$$
\text { Prime } i=\left[\begin{array}{lllll}
3 & 5 & 7 & 11 & 13 \ldots
\end{array}\right]
$$

It was argued that such signal would be less affected by nonlinear distortion. However, some combinations of harmonics in the feedback path may generate a frequency seen in the input excitation. For example, $\left[29 \omega_{o}-19 \omega_{o}-\right.$ $7 \omega_{o}$ ] will create a component at $3 \omega_{0}$. To overcome this, selection of No Interharmonic Distortion (NID) is proposed by (Evans, C. and Rees, D 2000a). It involves selecting a harmonic vector $\mathbf{i}$ so that, for the smallest possible maximum value of $i$ the relation (11) holds

$$
s=\sum_{\substack{m_{1}=-F \\ m_{1} \neq 0}}^{F} \sum_{\substack{m_{2}=-F \\ m_{2} \neq 0}}^{F} \ldots \sum_{\substack{m_{n}=-F \\ m_{n} \neq 0}}^{F} \sum_{p=1}^{n} i\left(m_{p}\right) .
$$

Resulting harmonic vector is

$$
N I D i=\left[\begin{array}{lllll}
1 & 5 & 13 & 29 & 49 \ldots
\end{array}\right]
$$

We have used this NID vector to reduce the effect of nonlinear distortion.

\subsection{No Interfering Distortion Signal with Optimized Crest Factor}

The crest factor $(C F)$ describes compactness of a multi-sine signal $u(t)$ in time domain with a specified amplitude spectrum. The crest factor of a signal $u(t)$ is defined as:

$$
C F=\frac{\|u(t)\|_{\infty}}{\left.\|u(t)\|\right|_{2}}
$$

i.e. the ratio of its peak value to its power. This problem is considered in the case where $\mathrm{u}(\mathrm{t})$ is a multisine with given amplitudes $A_{i}$. The power of $\|\mathrm{u}(\mathrm{t})\|_{2}$ does not depend on the phases $\phi_{\mathrm{i}}$, and so the crest-factor optimization problem is equivalent to choosing appropriate phase for each frequency to minimize ||$u(t) \mid \|_{\infty}$. This problem is highly nonlinear, nonconvex and non-smooth. There is no known algorithm that guarantees a global minimum, except in the simplest case where a search over all possible phases is feasible. Different algorithms for crest factor minimization have been reported, the most efficient and common being the $L_{\infty}$ algorithm based on a generalization of Polya's algorithm (Guillaume et al., 1991). The crest factor is thus the ratio of the $L_{\infty}$ norm and the $L_{2}$ norm where the general $L_{p}$ norm of the function $u(t)$ over the interval $[0 ; \mathrm{T}]$ is defined as

$$
L_{p}(u)=\left[\frac{1}{T} \int_{0}^{T}|u(t)|^{p} d t\right]^{\frac{1}{p}}
$$

Optimizing the crest factor for NID selected signal make a proper test signal for the identification of the systems with friction nonlinearity. Simulation produces some interesting results, which is presented in next section. In this paper we have used the same concept that (Solomou et al. 2002) propose to minimize the CF. The main idea of this method is that to achieve CF minimization by selecting their relative harmonic phases to reduce the error introduced by a cubic nonlinearity.

\section{SIMULATION RESULTS}

We use Mathwork Simulink as the simulation environment to demonstrate the effectiveness of the proposed algorithm. The target described in section 2.3 has been used and the excitation signal used is NID with crest factor optimized, presented in previous section. Signals are sampled at the rate of $2000 \mathrm{~Hz}$ and simulation runtime is $4.096 \mathrm{sec}$. So two periods of the excitation signal contains 8192 samples. Simulation is carried out with input amplitudes selected as [1 235 100] and for each of these amplitudes a perturbation of 0.5 volt is used for input pair differential calculation. Figure 5 [top] shows the magnitude plot of frequency response with the proposed scheme whereas results obtained using conventional method are shown in Figure 5 [bottom]. It is clear that the proposed differential input with NID signal produces consistent result for all levels of input amplitude. On the contrary, the responses vary with input amplitude when conventional approach is used (Figure 5 [bottom]). With the proposed method, signal amplitude of 2 volts and above gives the best linear approximation of the system. On the other 
hand, conventional approach gets the best approximation at 100 volt input excitation only (Figure 5 [bottom]).


Figure 5: Simulation result (magnitude plot)

Figure 6 shows frequency spectrum of the input $u_{2}-u_{1}$ and the corresponding output $v_{2}-v_{1}$ of the system for input excitation magnitude of 3-3.5. It is clearly evident that interfering harmonics are very small and almost negligible at the output.
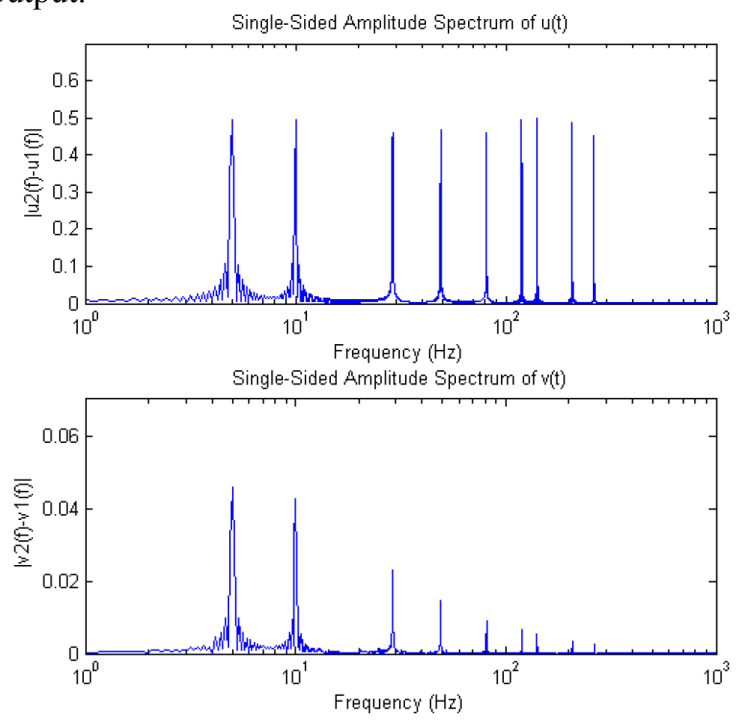

Figure 6: Spectrum of $\mathrm{u}$ and $\mathrm{v}$ for the proposed scheme

\section{EXPERIMENTAL RESULTS}

Figure 7 shows the experimental setup used to measure frequency response of the voice coil motor (VCM) of a 3.5" HDD. It consists of a Dynamic Signal Analyzers (DSA), a Laser Doppler Vibrometer (LDV) and a VCM power amplifier and the device under test (DUT) is a 3.5" HDD with a reflector mounted in its actuator head. The DSA is used for generating excitation signal as well as measurement of response. Acquisition of output signal is carried out using a high-performance, high-accuracy analog I/O device (NI PXI 4461). This board has two digital to analog converters (DAC) and two analog to digital converters (ADC), only one ADC is used. Each channel has its own sigma-delta converter with 24bits of resolution and a maximum sampling rate of $204.8 \mathrm{k}$ samples/s. The excitation signal and the LDV output are fed to two channels of the DSA. The magnitude and phase of the LDV output are compared to the measured stimulus signal to calculate the FRF. The velocity of the HDD's head is measured using the LDV (OFV 3001) with LDV range set at $25 \mathrm{~mm} / \mathrm{s} / \mathrm{V}$ during all experiments.

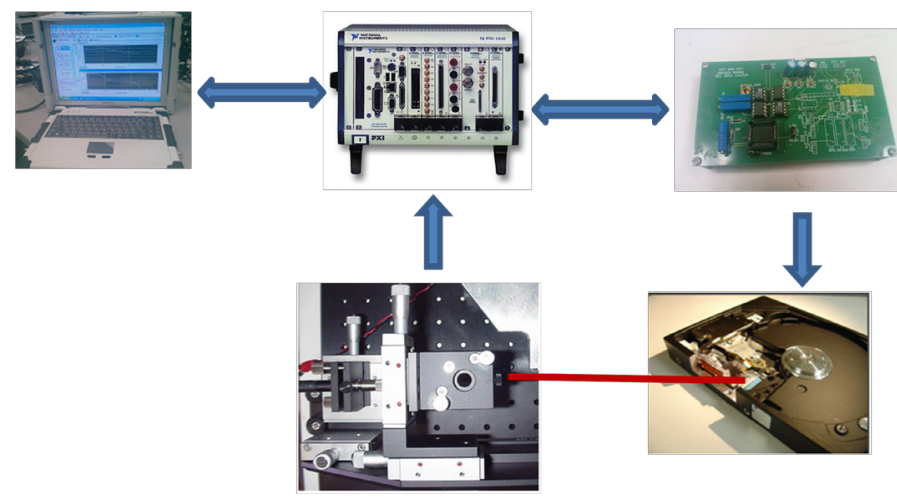

Figure 7: Block diagram of experimental setup

For all measurements, the FRF is computed for frequency range from $10 \mathrm{~Hz}$ to $1000 \mathrm{~Hz}$ with frequency spacing of 3.051 $\mathrm{Hz}$, resulting in an effective frequency resolution of $1.5 * 3.051$ $\mathrm{Hz}$ due to Hanning weighting of both channels. Every FRF is calculated using linear averaging of 20 data blocks of 327.7 $\mathrm{ms}$, acquired with free run averaging and the averaging mod is RMS averaging. The sampling frequency is $50 \mathrm{kHz}$.

Figure 8(a) shows the measured FRF of the system using conventional linear system identification method. Effect of friction is clearly evident in the magnitude plots of FRF. The response depends on the amplitude of input excitation. With prime harmonics multi-frequency excitation, perturbation of 2 $\mathrm{mV}$ is used for all amplitude levels. FRFs measured using this approach show consistent results for all amplitude levels, except $5 \mathrm{mV}$ [Figure 8(b)]. Estimated FRF is quite robust against changes in input amplitude.

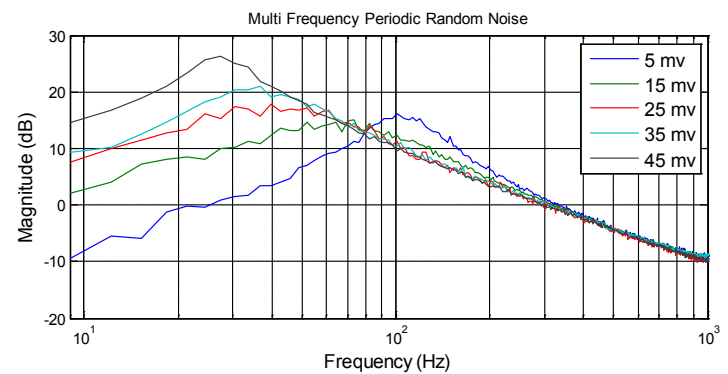

Figure 8(a): Measured response (conventional method) 


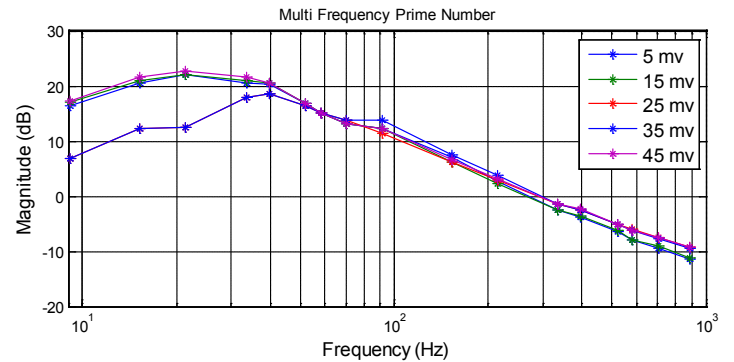

Figure 8(b): Measured response (proposed method)

\section{CONCLUSION}

Extension of linear techniques toward non-linear system analysis is studied in this paper. An improved technique is proposed to estimate the best linear model of a system with friction. Proposed method includes design of a multi sinusoidal input excitation with no interfering distortion at the input frequencies. After choosing appropriate frequency components, optimum phase of each frequency of input signal is obtained such that input crest factor is minimized. Simulation result shows that use of differential signal improves accuracy of the identified model and a linear model is found that is independent of input amplitude. Proposed method is verified experimentally.

\section{ACKNOWLEDGEMENT}

This work is supported by research grant from National Research Foundation (NRF) under CRP Award No. NRFCRP-4-2008-03

\section{REFERENCES}

Abramovitch, D., Feei Wang, and Franklin G. (1994). "Disk drive pivot nonlinearity modeling. I. Frequency domain," in American Control Conference., Baltimore, MD, vol. 3, 2600-2603.

Byun, Y. et al., (1997). Pivot nonlinearity in disk drive rotary actuator: measurement and modeling. Magnetics, IEEE Transactions on, 33(5), 2635-2637.

Evans, C. and Rees, D. (2000a). Non-linear Distortions and Multisine Signals - Part II: Minimizing the Distortion. IEEE Transactions on Instrumentation and Measurement, 49(3), 602-609.

Evans, C. and Rees, D. (2000b). Non-linear Distortions and Multisine Signals - Part II: Measure the Best Linear Approximation. IEEE Transactions on Instrumentation and Measurement, 49(3), 610-616.

Feei Wang et al., (1994). Disk drive pivot nonlinearity modeling. II. Time domain. In American Control Conference, (1994). American Control Conference, 1994. Vol. 3, 2604-2607.

Guillaume, P., J. Schoukens, R. Pintelon and I. Kollar (1991). Crest-factor minimization using nonlinear chebyshev approximation methods. Instrumentation and Measurement, IEEE Transactions. 40, 982-989.

Hensen, R., van de Molengraft, M. \& Steinbuch, M., (2002). Frequency domain identification of dynamic friction model parameters. Control Systems Technology, IEEE Transactions on, 10(2), 191-196.

Schoukens J., Pintelon R., Dobrowiecki T., Rolain Y., (2005a) Identification of linear systems with nonlinear distortions, Automatica, vol. 41, no. 3, 491-504.

Schoukens J.,Swevers J., Paduart J., Vaes D., Smolders K., Pintelon R., (2005b) Initial estimates for block structured nonlinear systems with feedback. International Symposium on Nonlinear Theory and its Applications, , pp. 622-625.

Kemao Peng et al. (2005), Modeling and compensation of nonlinearities and friction in a micro hard disk drive servo system with nonlinear feedback control, IEEE Transactions on Control Systems Technology 13(5): 708721.

Mueller, J., Antao, B. \& Saleh, R., (1996). A multifrequency technique for frequency response computation with application to switched-capacitor circuits with nonlinearities. Computer-Aided Design of Integrated Circuits and Systems, IEEE Transactions on, 15(7), 775790.

Solomou, M., Evans, C. \& Rees, D., (2002). Crest factor minimization in the frequency domain. Instrumentation and Measurement, IEEE Transactions on, 51(4), 859865.

Sherif, H.A. and Bassioni, A.S (1994). Non-linear identification of mechanical systems with dynamic dry friction. Journal of Sound and Vibration, 178(4), 513533.

Nagamune, N., Huang,X., and Horowitz, R., (2010) "Robust Control Synthesis Techniques for Multirate and Multisensing Track-Following Servo Systems in Hard Disk Drives," Journal of Dynamic Systems, Measurement, and Control 132(2): 021005.

Chen, Y.Y, Huang P.Y., and Yen, J.Y., (2002) "Frequencydomain identification algorithms for servo systems with friction," Control Systems Technology, IEEE Transactions on 10(5): 654-665. 